\title{
The application of a hierarchical Bayesian spatiotemporal model for forecasting the SAA trapped particle flux distribution
}

\author{
WAYAN SUPARTA* and GusRIZAL \\ Space Science Centre (ANGKASA), Institute of Climate Change, Universiti Kebangsaan Malaysia, \\ 43600 Bangi, Selangor Darul Ehsan, Malaysia. \\ *Corresponding author.e-mail: wayan@ukm.edu.my
}

We implement a hierarchical Bayesian spatiotemporal (HBST) model to forecast the daily trapped particle flux distribution over the South Atlantic Anomaly (SAA) region. The National Oceanic and Atmospheric Administration (NOAA)-15 data from 1-30 March 2008 with particle energies as $>30 \mathrm{keV}$ (mep0e1) and >300 keV (mep0e3) for electrons and 80-240 keV (mep0p2) and > $6900 \mathrm{keV}$ (mep0p6) for protons were used as the model input to forecast the flux values on 31 March 2008. Data were transformed into logarithmic values and gridded in a $5^{\circ} \times 5^{\circ}$ longitude and latitude size to fulfill the modelling precondition. A Monte Carlo Markov chain (MCMC) was then performed to solve the HBST Gaussian Process (GP) model by using the Gibbs sampling method. The result for this model was interpolated by a Kriging technique to achieve the whole distribution figure over the SAA region. Statistical results of the root mean square error (RMSE), mean absolute percentage error (MAPE), and bias (BIAS) showed a good indicator of the HBST method. The statistical validation also indicated the high variability of particle flux values in the SAA core area. The visual validation showed a powerful combination of HBST-GP model with Kriging interpolation technique. The Kriging also produced a good quality of the distribution map of particle flux over the SAA region as indicated by its small variance value. This suggests that the model can be applied in the development of a Low Earth Orbit (LEO)-Equatorial satellite for monitoring trapped particle radiation hazard.

\section{Introduction}

The development of a Low Earth Orbit (LEO)Equatorial inclination satellite is important for equatorial countries like Malaysia. This development will cover Malaysia's region more than the polar satellite does. However, the equatorial satellites certainly face more trapped particle radiation hazard than the polar satellites because they pass through the South Atlantic Anomaly (SAA) more frequently. In fact, for LEO satellites, the inner radiation belt where SAA is included, are a major factor for satellite anomalies (Horne et al. 2013). To help overcome this problem, this study attempts to forecast the distribution of trapped particles in the equatorial region, especially SAA.

In the current inner radiation trapped particle modelling, most models are based on physicsmagneto hydrodynamics (MHD) frame, such as SWMF/BATS-R-US with RCM (Tóth et al. 2005), Fok Ring Current (Fok et al. 2001), Plasmasphere (Pierrard et al. 2009), CIMI (Fok et al. 2011). The most used and the stable one is the AE-8/AP-8 (Vette 1991a, b), which runs in a statistical modelling framework.

Keywords. Forecasting; SAA; trapped particle; hierarchical Bayesian; spatiotemporal. 
In this work, we employ a statistical model, named Hierarchical Bayesian spatiotemporal (HBST) Gaussian process (GP) model for forecasting purpose. The uniqueness of our model is that it can be employed for both electrons and protons at different levels of solar activity conditions by using the geographic coordinates and dynamic data. At this time, our model is specifically applied for SAA region and valid for the positions of NOAA satellites altitude $(\sim 120$ to $\sim 800 \mathrm{~km}$ ). In that situation, we chose March 2008 as our model input. The purpose is to examine the applications of our model on a quiet day before being employed on a severe day when a major solar event happened. The results obtained by the model are then interpolated with a Kriging technique to figure the geographic distribution map of the trapped particle in the SAA region.

The following section summarizes the data available for analysis and HBST-GP model. In section 3, the analysis and discussion of both statistical and visual validation are presented. A brief summary and discussion of future works are given in section 4.

\section{Methodology}

\subsection{NOAA/POES data}

The NOAA/POES data are chosen in this study, acquired by the NOAA 15 satellite (http://satdat. ngdc.noaa.gov/sem/poes/data/avg/txt/). The detailed explanation for the data can be accessed through the National Aeronautics and Space Administration (NASA) - National Geophysical Data Center (NGDC) (http://satdat.ngdc.noaa. gov/sempoes/docs/readme_16s_ascii.txt). We select and sort the NOAA data at the area from $-90^{\circ}$ to $60^{\circ}$ of longitude and from $0^{\circ}$ to $-60^{\circ}$ of latitude to cover the SAA region. Once data is sorted, we move on to the next phase which is data cleaning. To stabilize the data distribution, we perform the logarithmic transformation to the cleaned data. Finally, the gridding process completes the data processing. The present model is used to calculate the trapped particle distribution of both electrons and protons, from low to high energy. The mep0e1 $(>30 \mathrm{keV})$ and mep0e3 $(>300 \mathrm{keV})$ represent the electrons whereas mep0p2 (80-240 keV) and mep0p6 (>6900 keV) represent the protons in the model.

The important step in this data processing is data gridding. NOAA 15 completes its orbit in about $1 \mathrm{hr}$ and $40 \mathrm{~min}$ (around 14 orbits in a day). Due to the fact that a point of observation cannot be observed in a long period of time. We have calculated that a point in a grid of an $1^{\circ} \times 1^{\circ}$ longitude and latitude has a two-month periodic observation, and this makes it impossible to do a forecast due to the lack of data. Therefore, gridding data is proposed to solve this problem. We propose to use the $5^{\circ} \times 5^{\circ}$ gridding system (see figure 1 ) .

Each data in a grid is averaged with its counterparts and the average value is put into the center of the grid. The new value and the new location then become a representative of the grid. We employed this procedure to have 360 grids of $5^{\circ} \times 5^{\circ}$ sizes over the SAA region. To minimize computing time, we decided to model and validate in 12 layers simultaneously from L1 to L12 as displayed in figure 1. Blue dot points in figure 1 represent the input points for the model fitting, whereas the red square cross points describe the validation points. Four points were selected randomly as the validation points in each layer.

The validation process is done by comparing the values in validation points obtained by the forecasting process with its original values obtained by observation. Data from 1-30 March 2008 has been

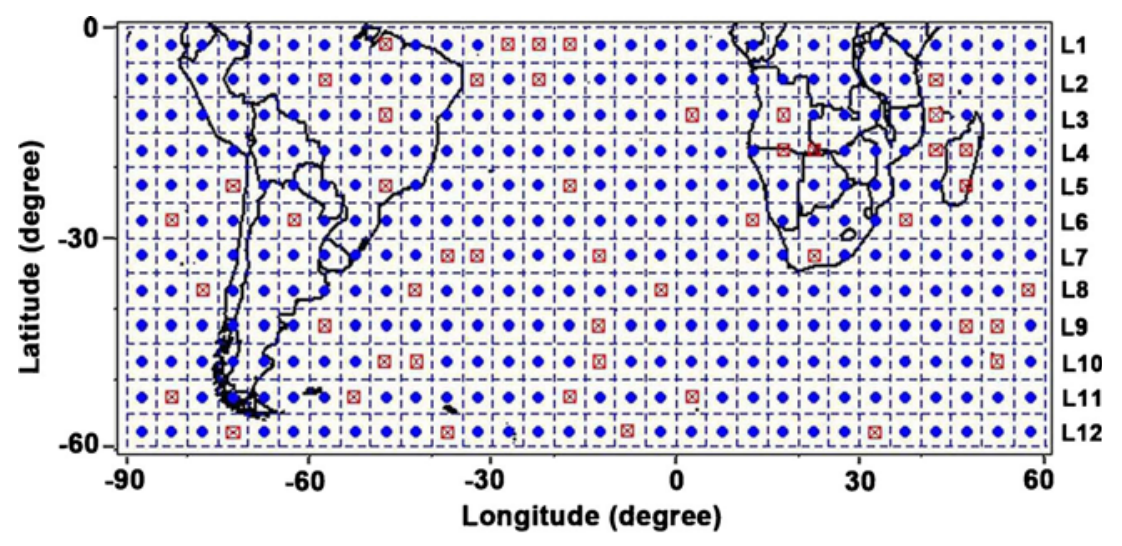

- grid center $\bullet$ fitting point $\otimes$ validation point

Figure 1. The HBST-GP fit and validation points. 
chosen to demonstrate the ability of the model. Our target is to forecast the flux values on 31 March 2008 with the help of the model.

\subsection{Hierarchical Bayesian spatiotemporal Gaussian process model}

The HBST model is a statistical modelling technique that deals with space time modelling using a Bayesian approach. They are used in the area of geosciences and environment, e.g., Riccio et al. (2006), Milliff et al. (2011) and Sahu and Bakar (2012). In this study, we are trying to apply this method in the space weather framework. According to Gelfand (2012), the HBST has a structure as shown below.

- First stage: Data model $[Z \mid E, \theta]$

- Second stage: Process model $[E \mid \theta]$

- Third stage: Parameter model $[\theta]$

where the data are defined by $Z$, the (hidden) process is defined by $E$, and the unknown parameters are specified by $\theta$.

First, we must define the generic notations used throughout this paper and the formula related to them (Sahu et al. 2013). Let $Z\left(s_{i}, t\right)$ denotes the logarithmic value of an SAA's particle flux at the site $s_{i}$ and time $t$, where $i=1, \ldots, n$ and $t=1, \ldots, T$. $E\left(s_{i}, t\right)$ is a true value corresponding to $Z\left(s_{i}, t\right)$. We put $T=30$ in this study to accommodate selected data from 1-30 March 2008 as the model input. We put 31 March 2008 as our forecasting target, notified as $T+1$. We choose to define both $Z$ and $E$ in vector notation as $Z_{t}=\left(Z\left(s_{i}, t\right), \ldots, Z\left(s_{n}, t\right)\right)^{\prime}$ and $E_{t}=\left(E\left(s_{i}, t\right), \ldots\right.$, $\left.E\left(s_{i}, t\right)\right)^{\prime}$. Thus, the HBST-GP model can be expressed as:

$$
\begin{gathered}
Z_{t}=E_{t}+\varepsilon_{t} \\
E_{t}=X_{t} \beta+\eta_{t}
\end{gathered}
$$

with $\varepsilon_{t}=\left(\varepsilon\left(s_{1}, t\right), \ldots, \varepsilon\left(s_{n}, t\right)\right)^{\prime} \sim N\left(0, \sigma_{\varepsilon}^{2} I_{n}\right)$ is an error process. Included in this term, $\sigma_{\varepsilon}^{2}$ is a nugget effect which is homogeneous in space and time whereas $I_{n}$ is the $n \times n$ identity matrix. The covariates influencing the $Z$ value is denoted by $X_{t}$ with an $n \times p$ matrix size, and $\beta=$ $\left(\beta_{1}, \ldots, \beta_{p}\right)^{\prime}$ is the $p \times 1$ vector of $X_{t}$, respectively. NOAA 15 data does not provide any covariate data for the trapped particle flux, so the intercept value is employed in equation (2). Furthermore, $\eta_{t}=\left(n\left(s_{1}, t\right), \ldots, n\left(s_{n}, t\right)\right)^{\prime} \sim N\left(0, \Sigma_{n}\right)$ is the spatially correlated error. Included in, $\Sigma_{n}=\sigma_{\eta}^{2} S_{n}=$ $\sigma_{\eta}^{2} \kappa\left(s_{i}, s_{j}, \phi, \nu\right)$ is a variance-covariance matrix with $i, j=1, \ldots, n$. Afterward, $\sigma_{\eta}^{2}$ is the site invariant common variance. $\kappa(. ; \phi, v)$ expresses the spatial correlation matrix with spatial decay $\phi$ and smoothness parameter $v$. The error parameters, $\varepsilon_{t}$ and $\eta_{t}$ are independent of each other. For future reference, $z$ denotes all observed data, $x$ denotes all covariates data and $E$ represents all augmented values. Finally, $\boldsymbol{\theta}$ is used to express all parameters used in our model $\left(\boldsymbol{\theta}=\left(E, \beta, \sigma_{\varepsilon}^{2}, \sigma_{\eta}^{2}, \emptyset\right)\right)$.

For forecasting purpose, the HBST-GP model at any observed point $\boldsymbol{s}_{i}$ for $T+1$ day is expressed as: follows:

$$
\begin{gathered}
Z\left(s_{i}, T+1\right)=E\left(s_{i}, T+1\right)+\varepsilon\left(s_{i}, T+1\right) \\
E\left(s_{i}, T+1\right)=x^{\prime}\left(s_{i}, T+1\right) \beta+\eta\left(s_{i}, T+1\right)
\end{gathered}
$$

with posterior predictive distribution of $Z\left(s_{i}, T+1\right)$ for a given $z$ is denoted by:

$$
\begin{aligned}
& \pi\left(Z\left(s_{i}, T+1\right) \mid \boldsymbol{z}\right)= \\
& \int \pi\left(Z\left(s_{i}, T+1\right) \mid \boldsymbol{\theta}, \boldsymbol{E}, E\left(s_{i}, T+1\right), \boldsymbol{z}\right) \\
& \quad \times \pi\left(E\left(s_{i}, T+1\right) \mid \boldsymbol{\theta}, \boldsymbol{z}\right) \pi(\boldsymbol{\theta}, \boldsymbol{E} \mid \boldsymbol{z}) \\
& \quad \times d E\left(s_{i}, T+1\right) d \boldsymbol{E} d \boldsymbol{\theta} .
\end{aligned}
$$

Finally, to summarize this method, we performed the following algorithm to predict the $Z\left(s_{i}, T+1\right)$ by using Monte Carlo Markov Chain (MCMC)Gibbs sampling with $j$ iterations:

1. Determine $\boldsymbol{\theta}^{(j)}$ and $\boldsymbol{E}^{(j)}, j \geq 1$ to form equation (5).

2. Determine $E^{(j)}\left(s_{i}, T+1\right)$ from $N\left(x_{T+1}^{\prime} \beta^{(j)}\right.$, $\left.\sigma_{\eta}^{2(j)}\right)$.

3. Finally, determine $Z^{(j)}\left(s_{i}, T+1\right)$ from $N\left(E^{(j)} \times\right.$ $\left.\left(s_{i}, T+1\right), \sigma_{\eta}^{2(j)}\right)$.

Once the value of $Z$ for $T+1$ day is obtained, then the result is displayed in a geographic map by using a Kriging interpolation technique. Details of the Kriging interpolation technique applied to the distribution of trapped particle over SAA region of our work can be found in Suparta et al. (2013).

\section{Result and discussion}

\subsection{Statistical validation}

The HBST-GP forecasting process was computed by using the spTimer (Bakar and Sahu 2013), a spatiotemporal package in $\mathrm{R}$ language ( $\mathrm{R}$ core team 2013 ). We set 13,000 as the number of iterations and discharge the first 3000 iterations to stride the starting effect. A statistical validation was then performed through three validation parameters, which are the root mean squared error (RMSE), mean absolute percentage error (MAPE) and bias 
(BIAS). Equations (6-8) describe the formulae of the validation parameters, where $n$ denotes the total number of observations, $z_{i}$ denotes the observed data, indexed by $i, \hat{z}_{i}$ denotes predicted value, and $\bar{z}$ and $\bar{z}_{p}$ are the mean of observation and prediction, respectively.

$$
\begin{aligned}
\text { RMSE } & =\sqrt{\sum_{i=1}^{n} \frac{\left(\hat{z}_{i}-z_{i}\right)^{2}}{n},} \\
\mathrm{MAPE} & =\frac{1}{n} \sum_{i=1}^{n}\left|\frac{\hat{z}_{i}-z_{i}}{z_{i}}\right|, \\
\mathrm{BIAS} & =\frac{1}{n} \sum_{i=1}^{n} \hat{z}_{i}-z .
\end{aligned}
$$
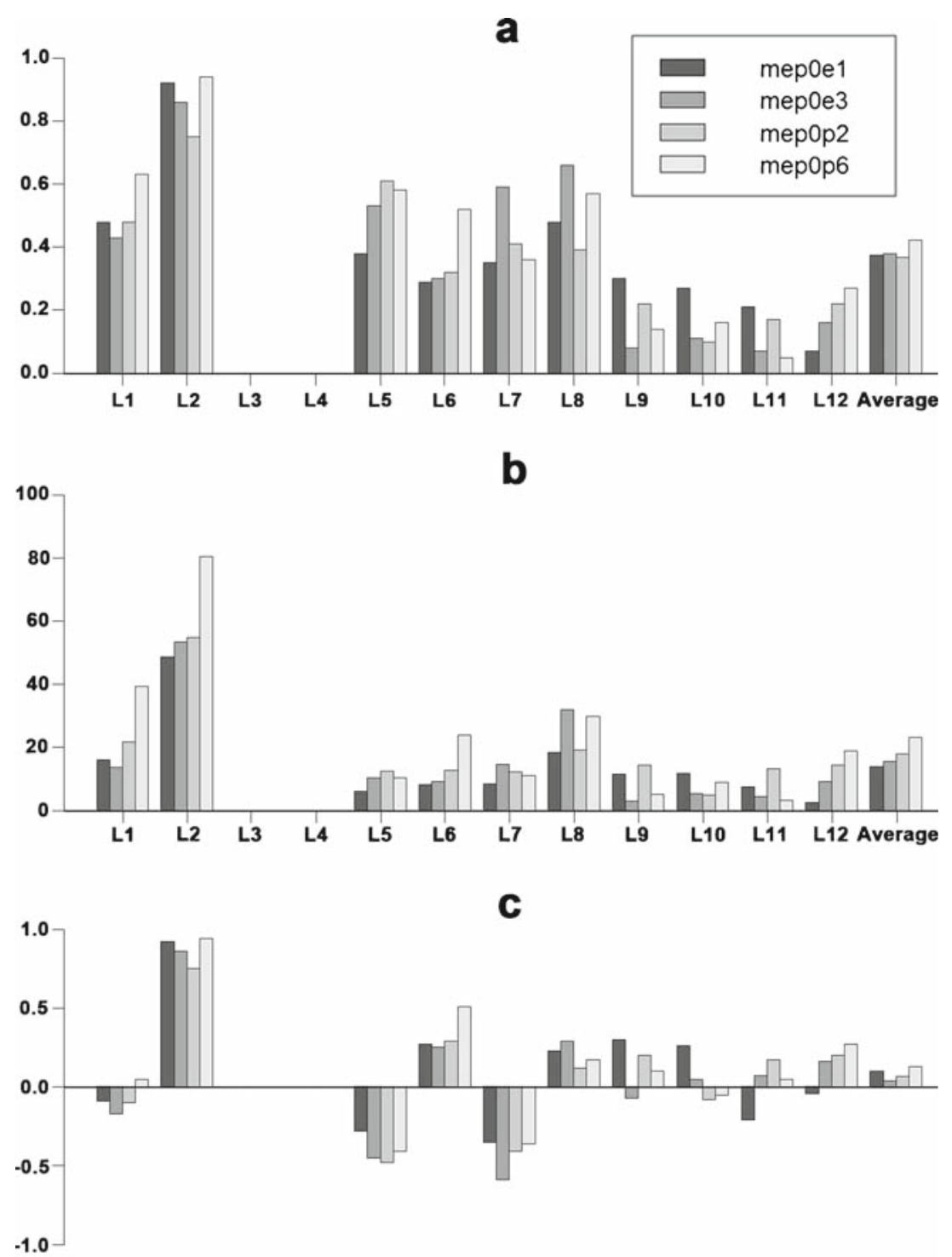

$\begin{array}{llllllllllll}\text { L1 } & \text { L2 } & \text { L3 } & \text { L4 } & \text { L5 } & \text { L6 } & \text { L7 } & \text { L8 } & \text { L9 } & \text { L10 } & \text { L11 } & \text { L12 Average }\end{array}$

Figure 2. Statistical validation results for (a) RMSE, (b) MAPE and (c) BIAS for all particles in every layer. The RMSE and BIAS units are in log flux (particles $/ \mathrm{cm}^{2} \mathrm{sec}$ str) whereas the MAPE unit is in percentage.
Figure 2 shows the results of the validation process. From figure 2(b), we can conclude that the forecast values have a high degree of forecasting since the MAPE average values produced are less than 25, i.e., 13.90, 15.53, 18.02 and 23.13, for mep0e1, mep0e3, mep0p2 and mep0p6, respectively. We also find that at the SAA dense flux area (L1 to L8 $/ 0^{\circ}$ to $-40^{\circ}$ of latitude) as seen in figure 4 , the trend of forecasting error has the tendency to increase. The errors start to rise at L2 which is a part of the SAA core area. Unfortunately, we have no validation results for L3 and L4 as the SAA core, since there was no data recorded at these validation points. The missing values occurred because the employment of single satellite data was not enough to fill up the entire grid in the gridding process. We also set a random selection of fitting 
and validation points to maintain the objectivity of our validation process. The high value of error in L1-L8 indicates that there was a high variation of flux value in those layers that make our model encountered a challenging task to perform forecasting. On the other hand, the small number of error in L9-L12 indicates the uniform distribution of flux value in those layers. We can also conclude that the variation value of mep0p6 at the SAA core is greater than the others, which produced highest MAPE value at L2 (80.53). In addition, figure 2(c) shows that our model mostly obtained overestimated forecasting results, except for L1, L5 and L7. Figures 2(a) and 2(c) also pointed that the maximum variance is approximately 1 (at L2). All these statistical analyses drive us to perform more treatments to deal with the SAA core flux variation.

\subsection{Visual analysis}

The visual analysis starts by understanding the NOAA 15 daily particle flux data and the NOAA flux distribution map, that for this analyzing process, we just give the map of mep0e1 for simplifying purpose (figure $3 \mathrm{a}$ and $\mathrm{b}$ ). As shown in figure 3(a), plotted NOAA 15 daily data reveal many unobserved areas in the entire globe, and that clarifies the missing data occurrence at L3 and L4 in the validation points (as explained in section 3.1). Figure 3(b) shows one of the particle flux distribution map generated by NOAA $15-18$ and METOP02 satellite data. From figure 3(b), we can find that the actual distribution of particle flux expanded beyond the SAA area. We also find that the flux particles are concentrated in polar regions.

Figure 4(a-d) represents the NOAA flux distribution map of mep0e1, mep0e3, mep0p2 and mep0p6, respectively. We only focus on the same area as taken in our model, to help us perform a visual validation. Figure $5(\mathrm{a}-\mathrm{d})$ is the result of forecasting process on 31 March 2008 after being interpolated, and consecutively represent mep0e1, mep0e3, mep0p2 and mep0p6. We utilized the $\mathrm{R}$ fields package (Furrer et al. 2013) to perform Kriging technique on our forecasting results. Figure $6(\mathrm{a}-\mathrm{d})$ provides the variance of Kriging on mep0e1, mep0e3, mep0p2 and mep0p6, respectively.
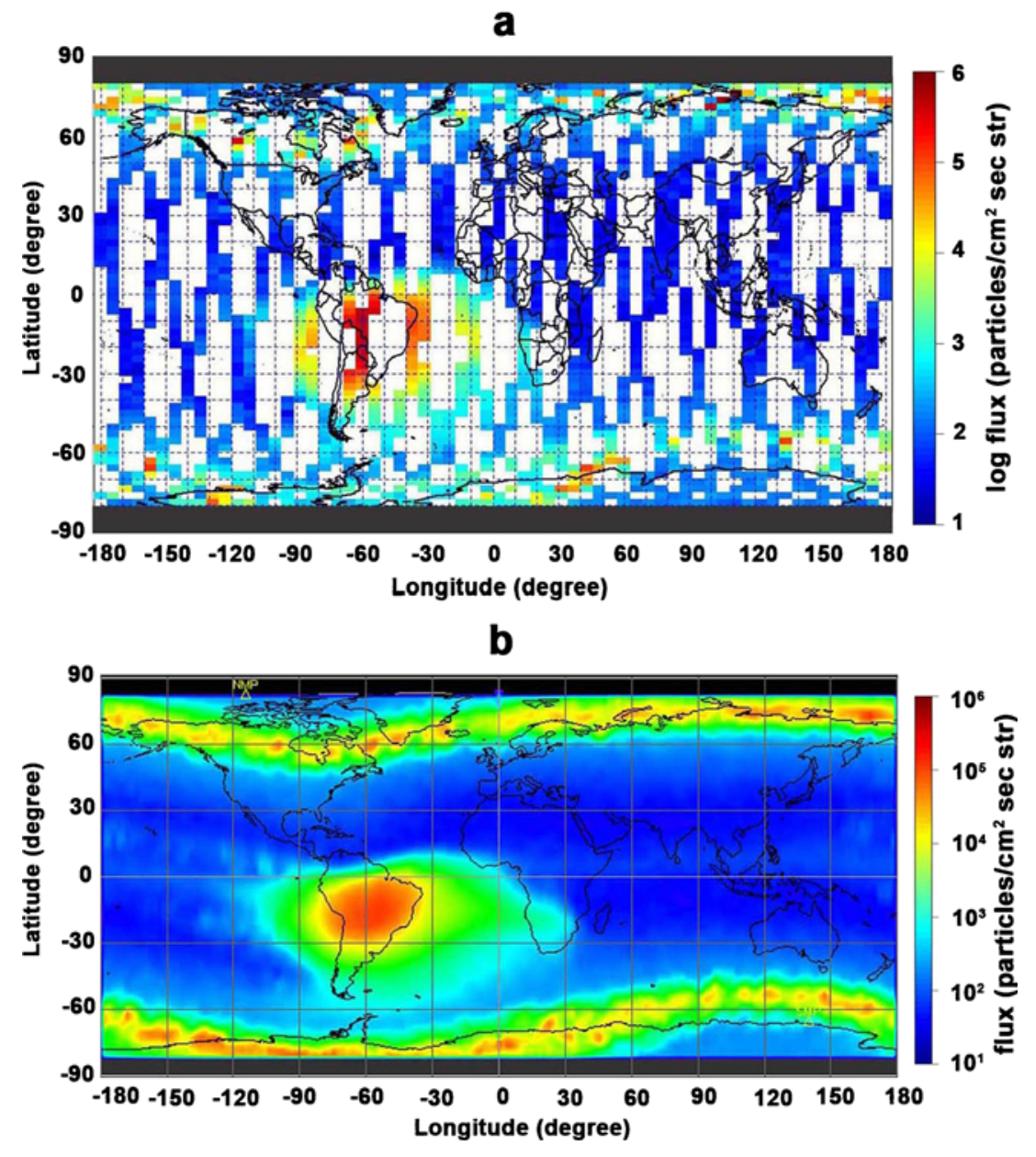

Figure 3. (a) NOAA 15 daily data plot of mep0e1 on 31 March 2008 and (b) NOAA's map of mep0e1 flux distribution from 28-31 March 2008 generated by NOAA 15-18 and METOPO2 satellite data. 
a

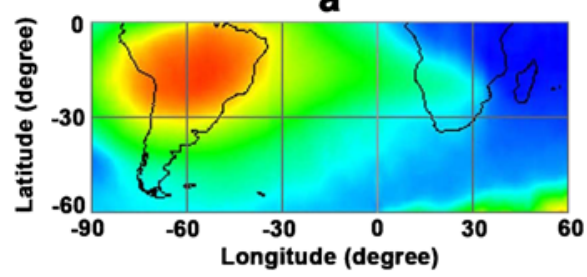

b

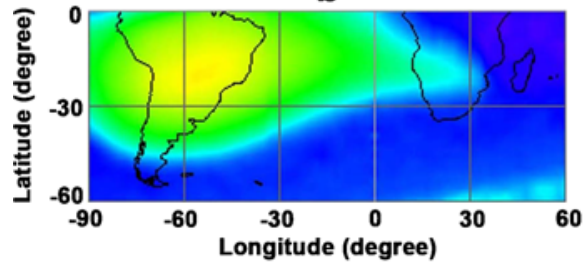

C

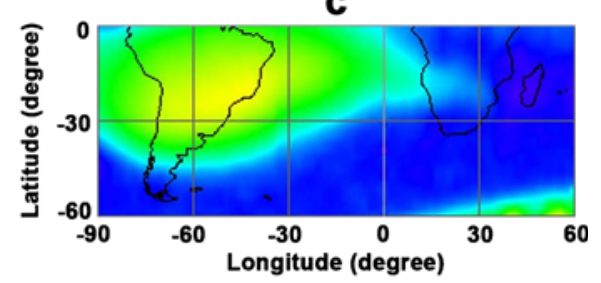

d

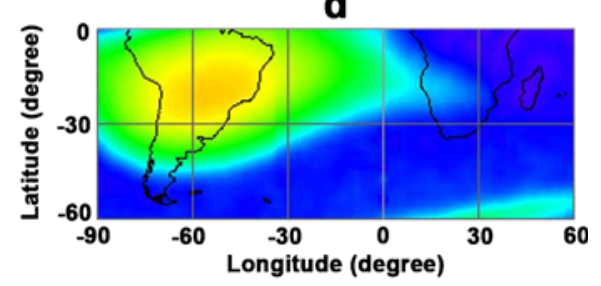

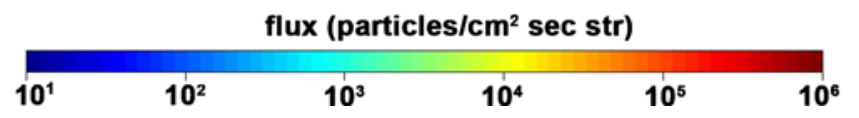

Figure 4. NOAA's distribution map for (a) mep0e1, (b) mep0e3, (c) mep0p2 and (d) mep0p6, on 31 March 2008, respectively.

a

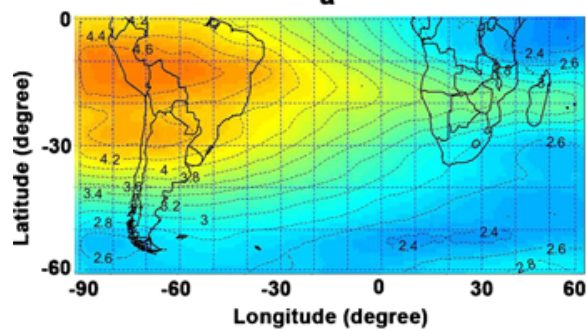

b

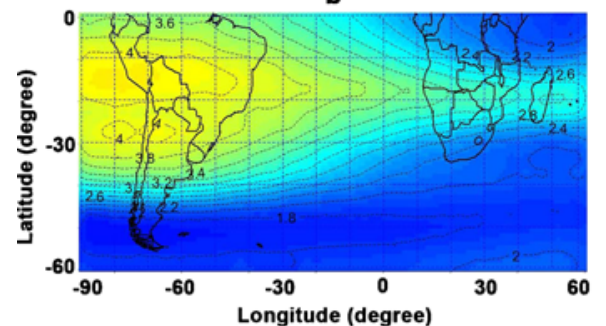

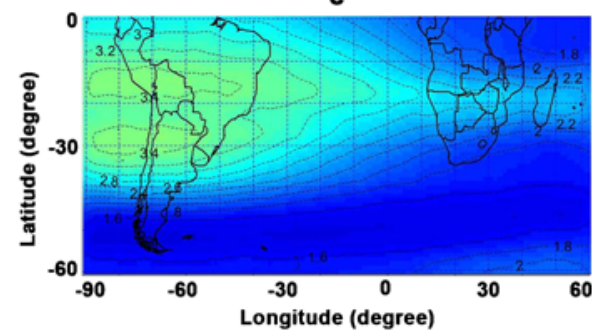

d

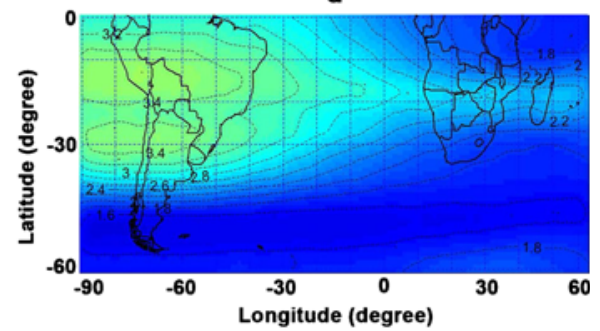

log flux (particles $/ \mathrm{cm}^{2}$ sec str)

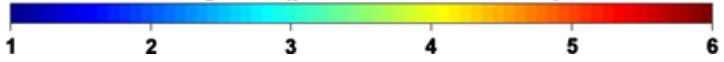

Figure 5. The estimations of Kriging interpolation for (a) mep0e1, (b) mep0e3, (c) mep0p2 and (d) mep0p6, on 31 March 2008.

From the comparison of figures 4 and 5 , we find that the distribution pattern is quite similar. We also put contour border lines in figure 5 to help us understand the distribution of particle flux in the $\mathrm{SAA}$ region. It is obvious from figure 5 that the high dense area in SAA is situated at around $-5^{\circ}$ to $-20^{\circ}$ of latitude (L2 to L4). We also notice that the bottom boundary of SAA is in around $-30^{\circ}$ to $-40^{\circ}$ of latitude (L6 to L8). These visual results strengthen our conclusion in section 3.1. Figure 5 also shows the powerful combination of HBST and Kriging interpolation technique, bringing about a conclusion that it can produce a good forecast as well as perform a proper interpolation to draw the whole distribution map over the SAA area. The NOAA's maps in figures 3(b) and 4 drawn by using five NOAA satellites are effective for 4 days distribution and their purpose is for nowcast prediction. On the other hand, our results obtained by only using NOAA 15 data are for purpose 

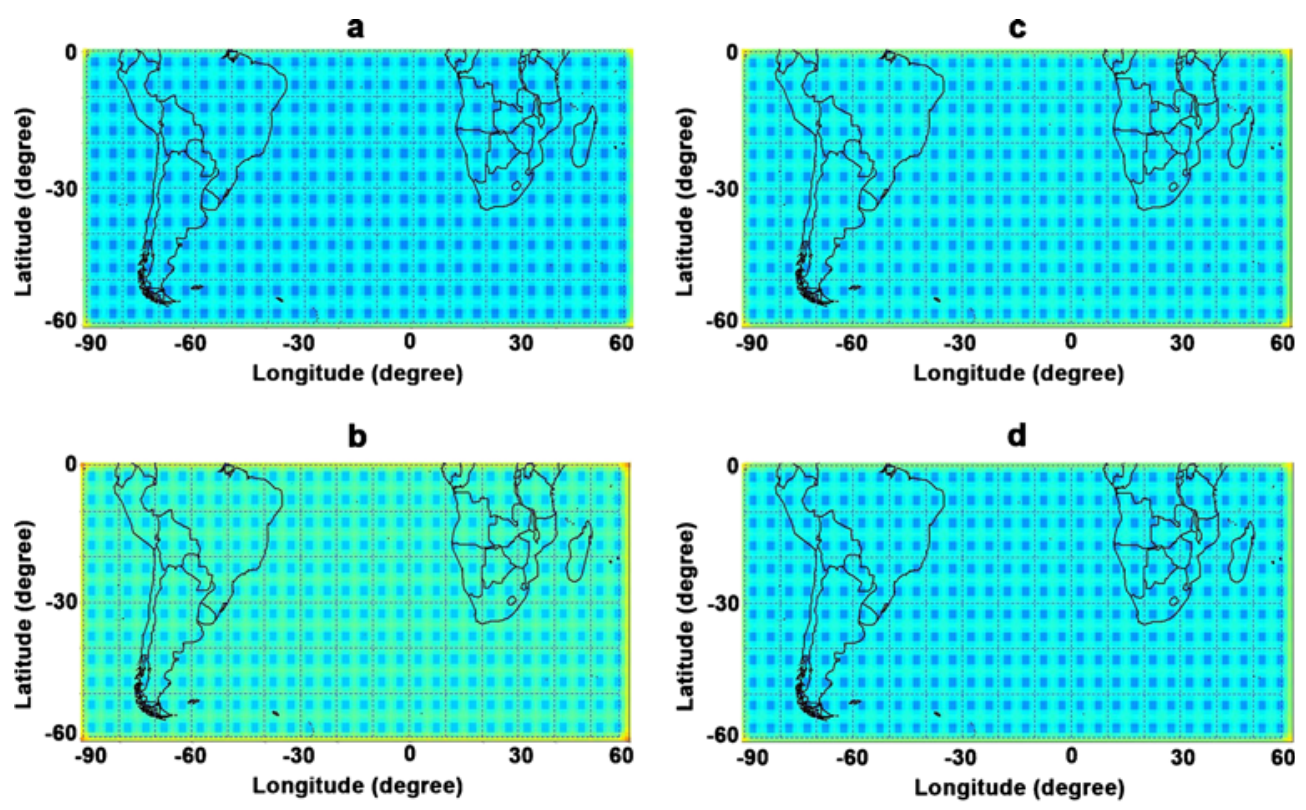

log flux (particles $/ \mathrm{cm}^{2}$ sec str)

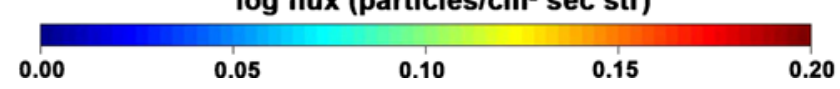

Figure 6. The variances of Kriging interpolation for (a) mep0e1, (b) mep0e3, (c) mep0p2 and (d) mep0p6, on 31 March 2008.

of daily forecast prediction. As reflected from figure 6, our Kriging quality produced a good interpolation, as pointed by a small variance (less than 0.10) for all particle fluxes. All visual analyses indicate that the Kriging technique is working properly to draw the distribution map of particle flux forecasted by HBST-GP model.

\section{Summary and future works}

This paper was successful to perform the preliminary work of LEO trapped particle's flux distribution forecast over the SAA region. The objective was obtained by applying HBST-GP model on NOAA 15 satellite data. We also achieved to figure the trapped particle flux distribution map by implementing the Kriging interpolation method on the geographic coordinate system. A statistical analysis showed good results and detected a great variability of particle flux values occurred in the SAA core area. This phenomenon is indicated by the high error of validation at the SAA core layers. Unfortunately, we cannot analyze the particle distribution at several areas of SAA core. This happened because there was a missing data occurrence at their validation points due to lack of data. In future work, we will propose to shrink the grid size into a $2^{\circ} \times 2^{\circ}$ of longitude and latitude gridding system. This effort has offered a better analysis for all layers distribution to minimize the high variation probability among the grid data. Another important point is to employ multiple NOAA satellite data, i.e., NOAA $15-18$. This intention is proposed to reduce the probability of missing data occurrence at validation points. As for the visual analysis, we can consider that Kriging technique worked properly for drawing the distribution map over the SAA region.

\section{Acknowledgements}

This study was supported by the Ministry of Science, Technology and Innovation Malaysia (MOSTI) under Science Fund 06-01-02-SF0808 grant. The authors sincerely thank National Oceanic and Atmospheric Administration (NOAA) USA for the data used. Special thanks to Mr. Muhammad Marizal for his advice on $\mathrm{R}$ programming.

\section{References}

Bakar K S and Sahu S K 2013 spTimer: Spatio-temporal Bayesian modeling using R; Technical Report, School of Mathematics, University of Southampton.

Fok M C, Wolf R A, Spiro R W and Moore T E 2001 Comprehensive computational model of the earth's ring current; J. Geophys. Res. 106 8417-8424, doi: 10.1029/2000JA000235.

Fok M C, Glocer A, Zheng Q, Horne R B, Meredith N P, Albert J M and Nagai T 2011 Recent developments in the radiation belt environment model; J. Atmos. Sol. Terr. Phys. 73 1435-1443. 
Furrer R, Nychka D and Sain S 2013 Fields: Tools for spatial data; $R$ package version 6.7.6, http:/CRAN. R-project.org/package $=$ fields.

Gelfand A E 2012 Hierarchical modeling for spatial data problems; Spatial Stat. 1 30-39.

Horne R B, Glaurent S A, Meredith N P, Boscher D, Maget V, Heynderickx D and Pitchford D 2013 Space weather impact on satellites and forecasting the earth's electron radiation belts with SPACECAST; Space Weather 11 169-186, doi: 10.1002/swe.20023.

Milliff R F, Bonazzi A, Wikle C K, Pinardi N and Berliner L M 2011 Ocean ensemble forecasting. Part I: Ensemble mediterranean winds from a bayesian hierarchical model; Quart. J. Roy. Meteorol. Soc. 137(657) 858-878.

Pierrard V, Goldstein J, Andre N, Jordanova V K, Kotova G A, Lemaire J F, Liemohn M W and Matsui H 2009 Recent progress in physics-based models of the plasmasphere; Space Sci. Rev. 145 193-229, doi: 10.1007/978-1-4419-1323-4_7.

R Core Team 2013 R: A language and environment for statistical computing; R Foundation for Statistical Computing, Vienna, Austria, ISBN 3-900051-07-0, http://www. R-project.org/.

Riccio A, Barone G, Chianese E and Giunta G 2006 A hierarchical bayesian approach to the spatio-temporal modeling of air quality data; Atmos. Environ. 40(3) 554-566, doi: 10.1016/j.atmosenv.2005.09.070.

Sahu S K and Bakar K S 2012 Hierarchical bayesian autoregressive models for large space-time data with application to ozone concentration modeling; Appl. Stochastic Models Bus. Ind. 28 395-415, doi: 10.1002/asmb.1951.

Sahu S K, Bakar K S and Awang N 2013 Bayesian forecasting using hierarchical spatio-temporal models with applications to ozone levels in the eastern United States; Technical Report, School of Mathematics University of Southampton, http://www.personal.soton.ac.uk/sks/ research/papers/sahubakarandwang2013.pdf.

Suparta W, Gusrizal, Ali M A M and Ahmad N 2013 Proc. 2013 IEEE Intern. Conf. Space Sci. Comm. (ICONSPACE), pp. 17-22, doi: 10.1109/IconSpace.2013. 6599425.

Tóth G et al. 2005 Space weather modeling framework: A new tool for the space science community; J. Geophys. Res. 110 A12226, doi: 10.1029/2005JA011126.

Vette J I 1991a The AE-8 trapped electron model environment; Annual Report, National Space Science Data Center (NSSDC)/World Data Center (WDC) 91-24.

Vette J I 1991b The NASA/national space science data center trapped radiation environment model program (19641991); Annual Report, National Space Science Data Center (NSSDC)/World Data Center (WDC) 91-29. 